\title{
Real-time monitoring of airborne cat allergen using a QCM-based immunosensor
}

\author{
Revised and resubmitted to: \\ Sensors and Actuators B: Chemical
}

September 5, 2013

DAVID R. P. MORRIS ${ }^{1}$, JULIEN FATISSON ${ }^{1,2}$, ADAM L. J. OLSSON ${ }^{1}$, NATHALIE TUFENKJI ${ }^{1}$, and ANDREA R. FERRO*,3

\author{
${ }^{I}$ Department of Chemical Engineering, Mc Gill University, \\ Montreal, Quebec H3A 2B2, Canada \\ ${ }^{2}$ current affiliation, Mechanical Engineering Department, École de technologie supérieure, \\ Montreal, Quebec, H3C 1K3, Canada \\ ${ }^{3}$ Department of Civil and Environmental Engineering, Clarkson University, \\ Potsdam, NY 13699 U.S.A.
}

*Corresponding Author. Phone: (315) 268-7649; Fax: (315) 268-7985; E-mail: aferro@clarkson.edu 


\begin{abstract}
The purpose of this study was to demonstrate the potential of real-time monitoring of airborne cat allergen powder using quartz crystal microbalance (QCM) technology. Monoclonal antibodies of cat allergen $(F e l d 1)$ were immobilized onto a gold-coated quartz crystal using a cysteamine self-assembled monolayer (SAM). The QCM responses exhibited negative frequency shifts characteristic of mass uptake onto the sensor surface when exposed to airborne cat allergen powder. The cat allergen powder was $0.01 \mathrm{wt} . \% \mathrm{Fel} d 1$; therefore, antigen-antibody binding occurred despite potential interference from dust particles. The absolute frequency shift increased linearly (log-log, $\left.R^{2}=0.968\right)$ over the immunosensor working range, from $5.2 \mathrm{ng} / \mathrm{L}$ to $1.6 \times 10^{5} \mathrm{ng} / \mathrm{L}$. Selectivity of the immunosensor between the cat allergen and a test dust was confirmed using SEM images and antibody affinity was confirmed by challenging an E. coli antibody-coated crystal with airborne cat allergen. Positive frequency shifts were observed with the QCM for both negative controls. The research demonstrates that immunosensors based on QCM technology may become a viable alternative to conventional methods of real-time monitoring of bioaerosols.
\end{abstract}




\section{KEYWORDS}

Quartz crystal microbalance (QCM); immunosensor; bioaerosol; cat allergen; self-assembled monolayer (SAM). 


\section{INTRODUCTION}

Bioaerosols are airborne particles of diverse biological origin that are ubiquitous in indoor and outdoor environments. Most bioaerosols are innocuous but many may cause adverse health effects in humans through irritation, allergic reaction, or infection [1,2]. The detection and monitoring of bioaerosols is generally conducted through a multi-step process that involves sample collection, separation of analytes and other particles, and analysis using optical, electrical or chemical methods. These processes are often cumbersome, time-consuming and expensive; hence, there is interest in developing integrated direct-reading bioaerosol samplers [1-5]. However, there are very few commercially available instruments that can distinguish between bioaerosols and other particles in real-time. The only such instrument, the ultraviolet aerodynamic particle sizer (UVAPS), can provide viable bioaerosol concentrations in real-time but it cannot identify species $[1,2]$.

An important category of bioaerosols are allergens, which are generally difficult to monitor in air and are usually collected from the settled dust using a vacuum method and brought to the laboratory for further analysis $[1,6]$. The most popular technique for allergen analysis is the commercially available enzyme-linked immunosorbent assay (ELISA). The ELISA technique relies on substrate transfer by enzyme-labeled antibodies to confirm allergen-antibody binding, a process that can be labor-intensive, time consuming, and financially constraining [4-7]. A modification of this technique is the multiplex array for indoor allergens (MARIA), which seemingly offers several practical advantages over ELISA, such as speed and enhanced detection range, but nonetheless relies on fluorescence labeling and cannot be used for real-time allergen monitoring $[1,6]$.

A technology that has received interest for the detection and monitoring of biological analytes is the quartz crystal microbalance (QCM) $[4,7,8]$. A mass rigidly attached to the surface of the QCM crystal causes a negative resonant frequency shift $(\Delta f)$ according to the Sauerbrey relation [9]: 


$$
\Delta f=-C_{f} \Delta m
$$

where $\Delta f(\mathrm{~Hz})$ is the frequency $(f)$ shift, $C_{f}\left(\mathrm{~Hz} \mathrm{~cm}^{2} / \mathrm{ng}\right)$ is the sensitivity factor of the crystal, and $\Delta m\left(\mathrm{ng} / \mathrm{cm}^{2}\right)$ is the change in mass on the crystal surface. The QCM approach does not require labeling and therefore only the frequency signal is needed to monitor the analyte. Hence, mass uptake onto the QCM crystal can be monitored in real-time. The QCM is also a sensitive instrument that can realize mass uptake on the order of $\mathrm{ng} / \mathrm{cm}^{2}[7]$. The analyte can be monitored through the use of a recognition material coated onto the crystal; e.g., the corresponding antibody of the allergen of interest. Simultaneous real-time monitoring of multiple analytes, i.e., multiplexing, is also easily accomplished by using multiple recognition elements. Therefore, the QCM-based biosensor approach is a viable alternative to conventional techniques due to its simplicity, sensitivity, selectivity and real-time monitoring capability $[3-5,7,8,10]$.

Monitoring of biological analytes using QCM technology has generally been confined to the aqueous phase, although some research has been conducted in the gas phase [3,5,7,8,10-12]. The first QCM-based gasphase antibody-based biosensor (immunosensor) was developed by Ngeh-Ngwainbi et al. They observed negative $f$ shifts $(\Delta f)$ when the antibody was exposed to parathion vapor, indicative of mass uptake onto the quartz crystal (QC). Some interference from other pesticides was observed, but the associated $f$ shifts were much smaller [13]. Others have suggested that the $f$ responses they obtained are not indicative of antigenantibody binding because no irreversible (baseline) $f$ shift was observed, which would be expected for a strong immunochemical reaction $[12,14]$. Rajakovic et al. investigated the adsorption of various pesticides (including parathion) and organic compounds to parathion antibody-coated QCs. They observed reversible $f$ shifts, which they suggested are indicative of non-specific chemisorption and physisorption to the QC, rather than selective antigen-antibody binding [14,15]. 
Some have suggested that the lack of water in gas-phase immunosensors could deactivate the immobilized antibody $[12,15]$. To mitigate these effects, some approaches have simulated the aqueous sensor environment for gas-phase monitoring. For instance, Stubbs et al. incorporated a hydrogel into their functionalized surface in their surface acoustic wave (SAW) device, which was used to detect fluorescent antigen vapor. These researchers reported that the hydrogel improved the stability of the immobilized antibodies, and they confirmed that antibody-antigen interaction occurred in the gas phase [12]. Owen et al. developed a QCM-based immunosensor for real-time detection of aerosolized influenza A virions using a SAM-based immobilized antibody technique. They used a nebulizer to simulate airborne particle concentrations from a sneeze or a cough while retaining moisture in the analyte. Baseline negative $f$ shifts were observed when the dry antibody-functionalized surface was exposed to pulses of virus suspensions without interference from other particles. Specific antigen-antibody binding was confirmed through atomic force microscopy (AFM) images and by demonstrating that no non-specific binding of the virus occurred with control antibodies [7]. Other QCM-based gas phase biosensors have used different techniques, such as simple physisorption of bioaerosols to clean gold QCs [3] and molecularly imprinted polymer (MIP) layers, where the analyte interacts with a similarly sized cavity, rather than by specific antigen-antibody interaction [16].

Herein, cat allergen was selected as a model biological analyte to appraise the use of a QCM-based immunosensor for a mixed bioaerosol suspension with other particles, i.e., dust. The main allergen produced by domestic cats (Felis domesticus) is the Fel $d 1$ glycoprotein, which causes a reaction in allergic humans and is also a risk factor of asthma development [6,17-19]. Cat allergen is ubiquitous in urban environments and it can be detected indoors in areas without a cat ever having been present [1,17-19]. The exposure pathway to allergens is primarily through air, although airborne concentrations are not often reported [1]. Luczynska et al. (1990) found that typical airborne Fel $d 1$ concentrations were between 2 and $20 \mathrm{ng} / \mathrm{m}^{3}$ in nine houses with cats, although the concentration increased significantly $\left(>40 \mathrm{ng} / \mathrm{m}^{3}\right)$ during domestic cleaning because of the large proportion $(60 \%)$ of small particles $(\leq 2.5 \mu \mathrm{m})$ [17]. Custovic et al. (1998) found a similar airborne $\mathrm{Fel}$ 
$d 1$ concentration range $\left(0.7-38 \mathrm{ng} / \mathrm{m}^{3}\right)$, but almost half of the particles $(49 \%)$ were very large $(>9 \mu \mathrm{m})$ [20]. Recently, Raja et al. (2010) analyzed 24-h $\mathrm{PM}_{10}$ filter samples from the homes of 10 subjects and found Fel $d$ 1 in concentrations ranging from $<0.1$ to $0.6 \mathrm{ng} / \mathrm{m}^{3}$ for airborne particles with aerodynamic diameters $\leq 10 \mu \mathrm{m}$ [21].

The purpose of this study is to appraise the use of QCM technology for real-time detection and monitoring of bioaerosols in air without separation of other particles. Monoclonal antibodies of Fel d 1 were immobilized onto a gold-coated crystal as the biorecognition element. The resultant $f$ shift demonstrates that the allergen particles readily bind to the antibody-functionalized surface despite potential interference from dust particles. Moreover, the QCM measurements are supported by SEM imaging of Fel $d 1$ antibody functionalized QCs challenged with cat allergen powder or test dust.

\section{EXPERIMENTAL METHODS}

\subsection{Reagents and Materials}

Fel $d 1$ particles were obtained from controlled milling of cat fur obtained from pet grooming establishments by the Indoor Environment Center at The Pennsylvania State University [22]. The milled allergen powder is approximately $100 \mu \mathrm{g}$ allergen per g powder (0.01 wt.\%), as determined by ELISA using Fel $d 1$ antibodies. This concentration is within the range of measured indoor Fel $d 1$ concentrations in homes in the United States of $0.01 \mu \mathrm{g} / \mathrm{g}$ to more than $1000 \mu \mathrm{g} / \mathrm{g}$ as determined by Arbes et al. (2004) from sampling 831 homes [18]. The particle number size distribution as determined by a Malvern Mastersizer 2000 (Worcestershire, UK) has a single particle diameter mode at $0.5 \mu \mathrm{m}$, while the particle volume size distribution has a mode diameter of $13 \mu \mathrm{m}$. These distributions are provided in Fig. 1a and 1b, respectively. Due to the 
limitations of the optical instrument when sizing non-spherical particles of various compositions over a wide range of sizes, the distributions shown in Fig. 1a and $1 \mathrm{~b}$ are considered to be approximate size distributions.
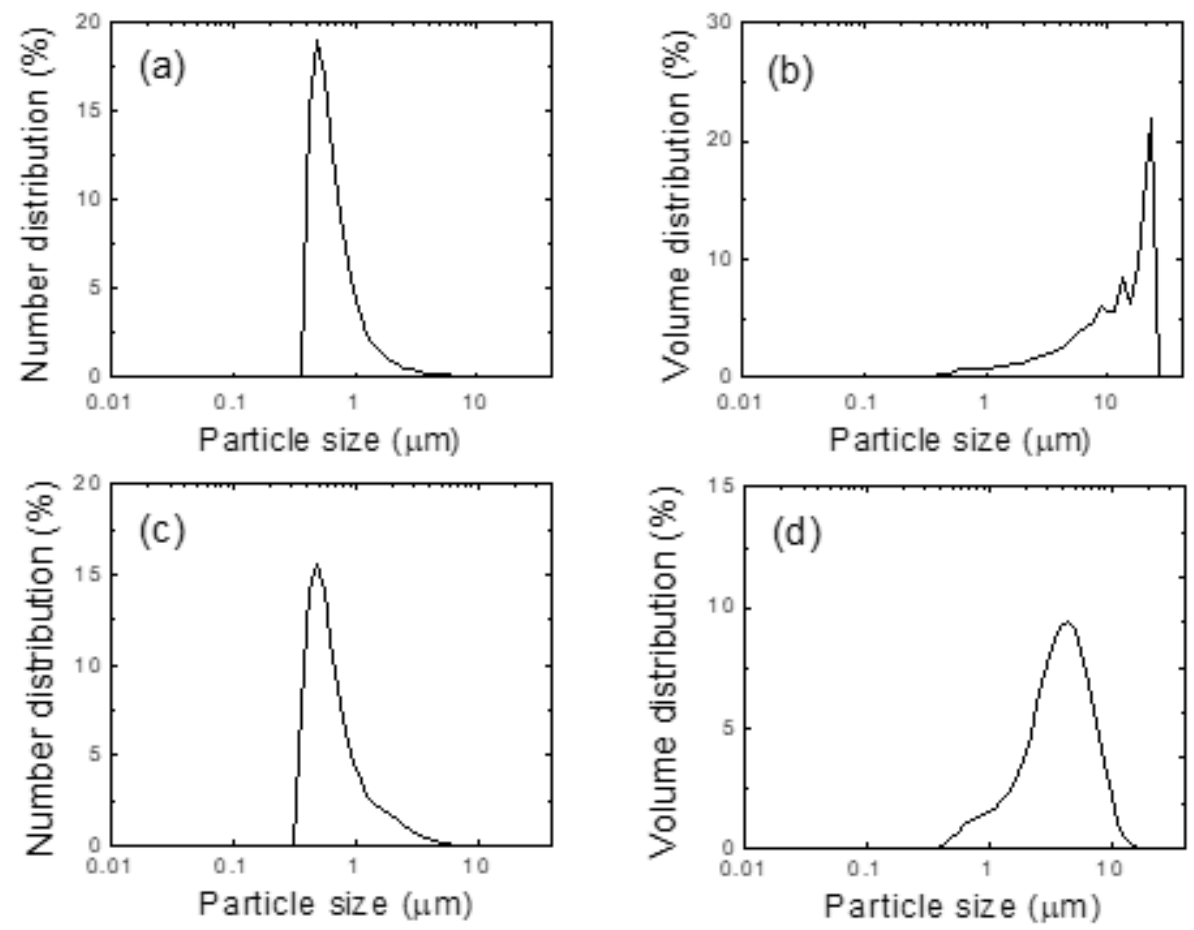

Fumed silica particles (CAB-O-SIL M-5, Cabot) with a mean aggregate particle diameter of 0.2-0.3 $\mu \mathrm{m}$ were added to the allergen powder (5 wt.\%) to reduce agglomeration and promote resuspension so that most (95\%) of the particle mass was cat allergen powder. Negative control experiments were conducted using ISO 12103-1, A1 Ultrafine Test Dust (Powder Technology Inc.), which contains 68-76 wt. \% $\mathrm{SiO}_{2}$ and has a similar particle number size distribution to $\mathrm{Fel} d 1$ as determined by a Malvern Mastersizer 2000 with a particle diameter mode at $0.5 \mu \mathrm{m}$ (Figure $1 \mathrm{c}$ and $1 \mathrm{~d}$ ).

Monoclonal antibodies specific to Fel $d 1$ were obtained from Indoor Biotechnologies (Charlottesville, VA). Cysteamine (Sigma-Aldrich), bovine serum albumin (BSA) (Sigma-Aldrich), and glutaraldehyde (Fisher) were used for surface modification of the gold-coated quartz crystals. Affinity purified polyclonal antibodies, specific to E. coli O157:H7 (Kirkegaard and Perry Laboratories), were also used to validate the 
immunosensor response. Denatured alcohol (Fisher Scientific), Hellmanex (Hellma), hydrogen peroxide (Fisher Scientific) and ammonia (LabChem Inc.) were used to clean the crystal surfaces after each experiment. Ultrapure deionized (DI) water was used to prepare all solutions.

\subsection{Surface Modification Procedure}

Clean, gold-coated QCM crystals (5 MHz, AT-cut, Inficon) were immersed in a $10 \mathrm{mM}$ cysteamine solution overnight at room temperature with ultra-high purity (UHP) nitrogen $\left(\mathrm{N}_{2}\right)$ bubbling through the solution. The crystals were rinsed with DI water and dried with UHP $\mathrm{N}_{2}$. The crystals were then rinsed with phosphate buffer saline (PBS) at $\mathrm{pH}$ 7.4. Subsequently, the crystals were immersed in a $2 \%$ solution of glutaraldehyde (which acts as a cross-linker molecule) in PBS for 1 hour. After rinsing the crystals with PBS to remove any unreacted glutaraldehyde, a $10 \mu \mathrm{g} / \mathrm{mL}$ solution of $\mathrm{Fel} d 1$ antibodies in PBS was deposited over the crystal surface for 90 min to allow time for the antibody to bind to the glutaraldehyde. After crosslinking, the crystal surfaces were rinsed with PBS and then left in contact with a $2 \%$ BSA solution in PBS, in order to block unreacted sites. After surface modification, the crystals were rinsed twice with PBS, twice with water, carefully dried under a gentle flow of UHP $\mathrm{N}_{2}$ and then mounted in the QCM crystal holder prior to conducting measurements [8].

\subsection{Apparatus}

The flowchart provided in Fig. 2a depicts the experimental setup. A small amount $(<5 \mathrm{~g})$ of cat allergen powder with 5 wt.\% fumed silica was placed in a 1-L, electrically grounded, cylindrical stainless steel resuspension chamber. The powder was resuspended by an incoming jet of HEPA-filtered room air at $4 \mathrm{~L} / \mathrm{min}$ via vacuum pump (BGI 400, $\pm 5 \%$, full scale (FS): $10 \mathrm{~L} / \mathrm{min}$ ). Airflow was measured using a rotameter (ShoRate, FS: $10 \mathrm{~L} / \mathrm{min})$. The powder was aspirated into the exposure chamber through a short $(5 \mathrm{~cm})$ inlet $(1 / 4 \mathrm{in}$. inner diameter (ID), path 1 in Fig. 2). The crystal apparatus was secured in an exposure chamber identical to 
the resuspension chamber and inverted to avoid gravitational settling of particles onto the QC surface (point 4 in Fig. 2). Short lengths $(<4 \mathrm{~cm})$ of $1 / 4$ in. ID Tygon ${ }^{\circledR}$ tubing were connected to the inlet and outlets inside the exposure chamber and aligned to reduce bypassing as well as to promote helical airflow, as shown in Fig. $2 b$ as trajectories 1,2 and 3. Turbulent flow was produced inside the chamber by the inlet, and eddies were formed by the sensor plate, which was located in the path of the airflow. Therefore, we expect that the allergen powder contacted the QC surface via both impaction and turbulent diffusion. Allergen binding to the antibody-coated gold surface was monitored using a QCM apparatus (Maxtek RQCM) with RQCM Data-logger software.
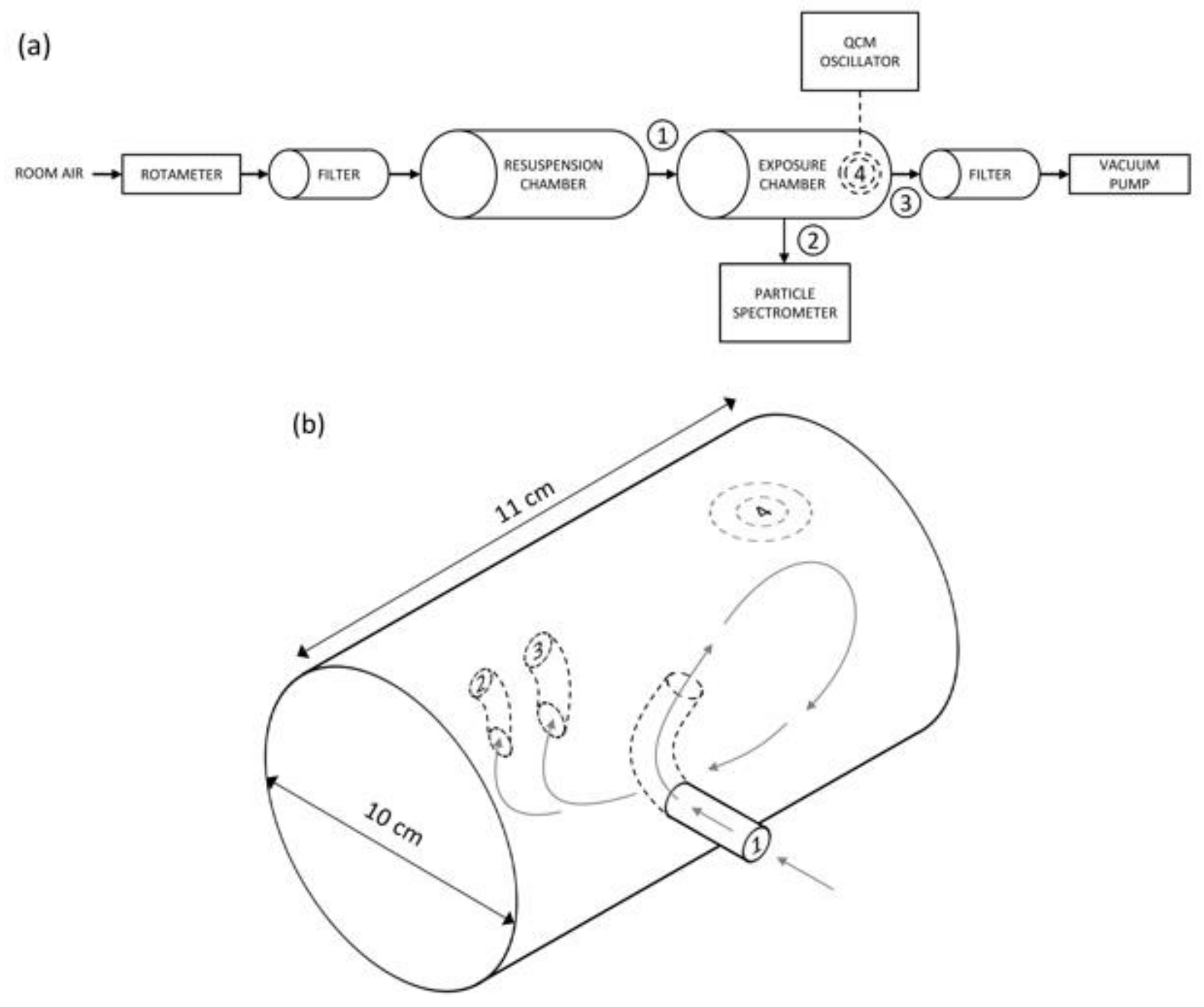
All experiments were conducted at ambient conditions. The relative humidity $(\mathrm{RH})$ in the exposure chamber was measured using a HOBO H8 data logger (Onset Computer Corp., $\pm 2.5 \%$ ). The particle concentration of Fel $d 1$ in the exposure chamber was measured using an optical particle counter (OPC, Grimm Aerosol Technik GmbH, Model 1.108), which samples air at 1.2 L/min. The OPC provides the size and number concentration of aerosol particles in real-time by light scattering of individual particles with a laser. The lower detection limit of the model that was used is $0.3 \mu \mathrm{m}$.

\subsection{Mass Concentration of Fel d 1}

Equation ((2) was used to estimate the particle mass concentration $(M)$ from the number concentration $(N)$ given by the particle spectrometer, where $\rho$ is the estimated particle density $\left(1.44 \mathrm{~g} / \mathrm{cm}^{3}\right), D_{p}$ is the geometric mean diameter for the size range $k$, size classes $k=1$ through $n$ correspond with the desired size range for $M$, and $a$ is an adjustment factor to account for the allergen dust being neither non-spherical nor optically identical to the calibration dust.

$$
M=a \sum_{k=1}^{n} N\left(\frac{\pi}{6} D_{p}^{3}\right) \rho=a M_{O P C}
$$

To determine a value for the adjustment factor $a$, the particle spectrometer and time-integrated $10 \mathrm{~L} / \mathrm{min}$ $\mathrm{PM}_{2.5}$ and $4 \mathrm{~L} / \mathrm{min} \mathrm{PM}_{10}$ single-stage particle impactor sampling devices (PEM 761-203B and 761-200A, SKC Inc.) were collocated in a $1 \mathrm{~m}^{3}$ chamber for several hours with resuspended Fel $d 1$ powder. The impactors were equipped with tared 37-mm Teflon filters. The particle mass concentration from the filter samples $M$ was determined by subtracting the difference of the blank corrected initial and final masses of the Teflon filters and dividing by the sampling time and flow rate. The ratio of the mass concentration measured by the filter samples $M$ and mass concentration estimated from the OPC from the collocation experiments MOPC (i.e., Equation ((2) solved for $a$ ) was used as an estimate for $a$. Values for $a$ using the Fel $d 1$ powder ranged from 0.8 to 1.2. 


\subsection{Scanning Electron Microscopy}

QCs were antibody-functionalized as described above (see section 2.2) and placed in the exposure chamber subjected to Fel $d 1$ powder or to test dust (negative control) under the same conditions as for any QCM experiment. QCs were then carefully removed from the exposure chamber and gold-palladium coated prior to observations using field emission gun scanning electron microscopy (FEG-SEM, Hitachi S-4700). An E. coli antibody-coated QC (additional negative control) was platinum-coated and observations were also made using FEG-SEM (Hitachi SU-70).

\section{RESULTS AND DISCUSSION}

\subsection{Characterization of the QCM Response}

The QCM response for antigen-antibody binding at various allergen concentrations is presented in Fig. 3 where the frequency $(f)$ shift is reported in Hz. The $f$ shifts of experiments conducted using cat allergen powder exhibit characteristic Sauerbrey-like behavior; namely, the negative difference between the fundamental and resonant frequency of the crystal $(\Delta f<0)$ typifies mass uptake onto the immunosensor by Equation $(1)$. Conversely, the $f$ shifts measured when the immunosensor is exposed to test dust are positive $(\Delta f>0)$, i.e., non-Sauerbrey-like behavior. Similar positive $f$ shifts were observed when bacterial (E. coli O157:H7) antibody-coated QCs were exposed to cat allergen powder at different concentrations $\left(2.2 \times 10^{2} \mathrm{ng} / \mathrm{L}\right.$ to $1.4 \times$ $\left.10^{3} \mathrm{ng} / \mathrm{L}, n=3\right)$. A representative result is shown in Fig. 3.

Positive $f$ shifts can be explained by a coupled resonance model, in which the oscillation of the sensor surface couples to the inherent resonant frequency of the particles. The inherent resonant frequency of the particle is determined by both its mass and contact point stiffness/bond strength. Strong interaction causes the particle to move in tandem with the oscillating surface, i.e., Sauerbrey-like behavior, whereas weak interaction 
allows the particles to oscillate freely over the surface, thereby inducing positive $f$ shifts [23-26]. Positive $f$ shifts have been observed in the gas $[16,24,25]$ and aqueous phases $[4,5,23,26]$ with weakly interacting species. For instance, Latif et al. found negative $f$ shifts when sesame antibodies were exposed to sesame proteins and positive $f$ shifts when exposed to almond proteins [26]. Jenik et al. observed positive $f$ shifts when non-specific species rolled across a QC surface [16]. Some have suggested that positive and negative $f$ shifts can even be used to distinguish between selective and non-selective interaction of analytes [16,26].

In sum, the positive $f$ shifts observed with the test dust and E. coli antibody-coated crystal are associated with weak adhesion forces whereas the negative $f$ shifts observed for the allergen samples correspond to strong adhesion forces. Weak interaction between cat allergen powder and the E. coli antibody-coated QC was expected as the antibody should have no cross-reactivity with Fel $d$. Further confirmation of the Sauerbreylike response of the QCM when tested with cat allergen powder can be obtained by examining the change in resistance of the resonator, which should be zero for a rigid mass with minimal damping [4]. The average measured change in resistance of the resonator after $30 \mathrm{~min}$ of $\mathrm{Fel} \mathrm{d} 1$ exposure was found to be minimal across the range of concentrations tested (Figure S1). Thus, the measured change in resistance confirms the Sauerbreylike behavior of the Fel $d 1$ antibody functionalized immunosensor. 


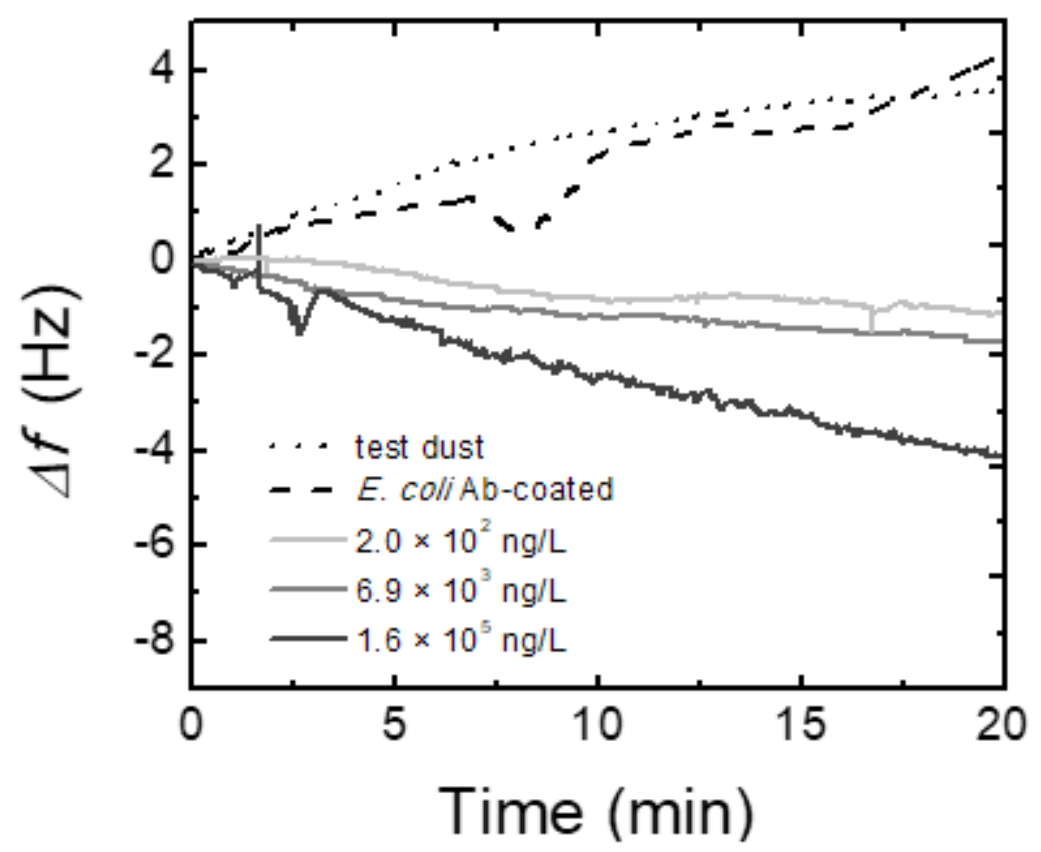

The magnitudes of the $f$ shifts are similar to those reported by Lee et al. (2008) [3], yet smaller than those reported by others $[7,13,15,16]$. These differences can be attributed to a number of different experimental factors, including: differences in particle size, mass, concentration and contact point stiffness; higher fundamental frequency $(9-10 \mathrm{MHz})$ of the QCs; use of different techniques, such as MIP and double-sided QCs; and much lower carrier gas flowrates $(<100 \mathrm{~mL} / \mathrm{min})$. Furthermore, the aerosols used in these other studies generally consisted solely of the bioanalyte (e.g., virus, pesticide or pollen) in inert carrier gas, i.e., there was no interference on the sensor from other particles such as the majority (>99.99 wt.\%) of the suspension in this study.

Water droplets in the carrier gas can cause interference by adsorbing onto the sensor surface. The RH and absolute humidity (AH) of the exposure chamber was monitored to determine if water had an effect on the QCM response. The RH and AH differences after 30 min were determined and compared with the immunosensor $f$ responses at different cat allergen concentrations. No correlation between them was discovered, which suggests that neither the $\mathrm{RH}$ nor the $\mathrm{AH}$ had an impact on the $f$ response (refer to supplementary information, Figure S2). Aside from water droplets, it is also possible that some particles in 
the dust may produce a positive artifact by sticking to the sensor (e.g., hydrophobic particles) [4]. SEM imaging of the QC directly following a QCM experiment was used to confirm that few dust particles attached onto the sensor surface (described in section 3.2 below).

\subsection{Validation of the Immunosensor}

As mentioned above, the QCM responses from test dust and E. coli antibody experiments exhibited positive $f$ shifts, representative of weak, non-specific interactions between the particles and antibodies. The clear contrast between the QCM response generated from the control experiments and those from the allergen powder suggest that the allergen particles are indeed forming a strong bond with the functionalized surface. To verify the hypothesis that selective antigen-antibody binding occurred, scanning electron microscopy (SEM) images were taken of the Fel $d 1$ antibody-coated surface after 30 min of exposure to the cat allergen and test dust (Figs. 4a and 4b). Images were also taken of the E. coli antibody-coated QC after 30 min of cat allergen powder exposure (Fig. 4c). Fig. 4a clearly shows that Fel $d 1$ particles readily attach onto the Fel $d 1$ antibody-functionalized surface. In contrast, no dust deposition (Fig. 4b) on the biorecognition surface was observed, even at higher magnification (images not shown). Therefore, the insignificant attachment of test dust onto the QC and thus the lack of non-specific binding of particles to the sensor surface were confirmed. Moreover, no cat allergen or dust deposition was observed on the E. coli antibody-coated surface (Fig. 4c), thereby confirming selectivity of the antibody. 

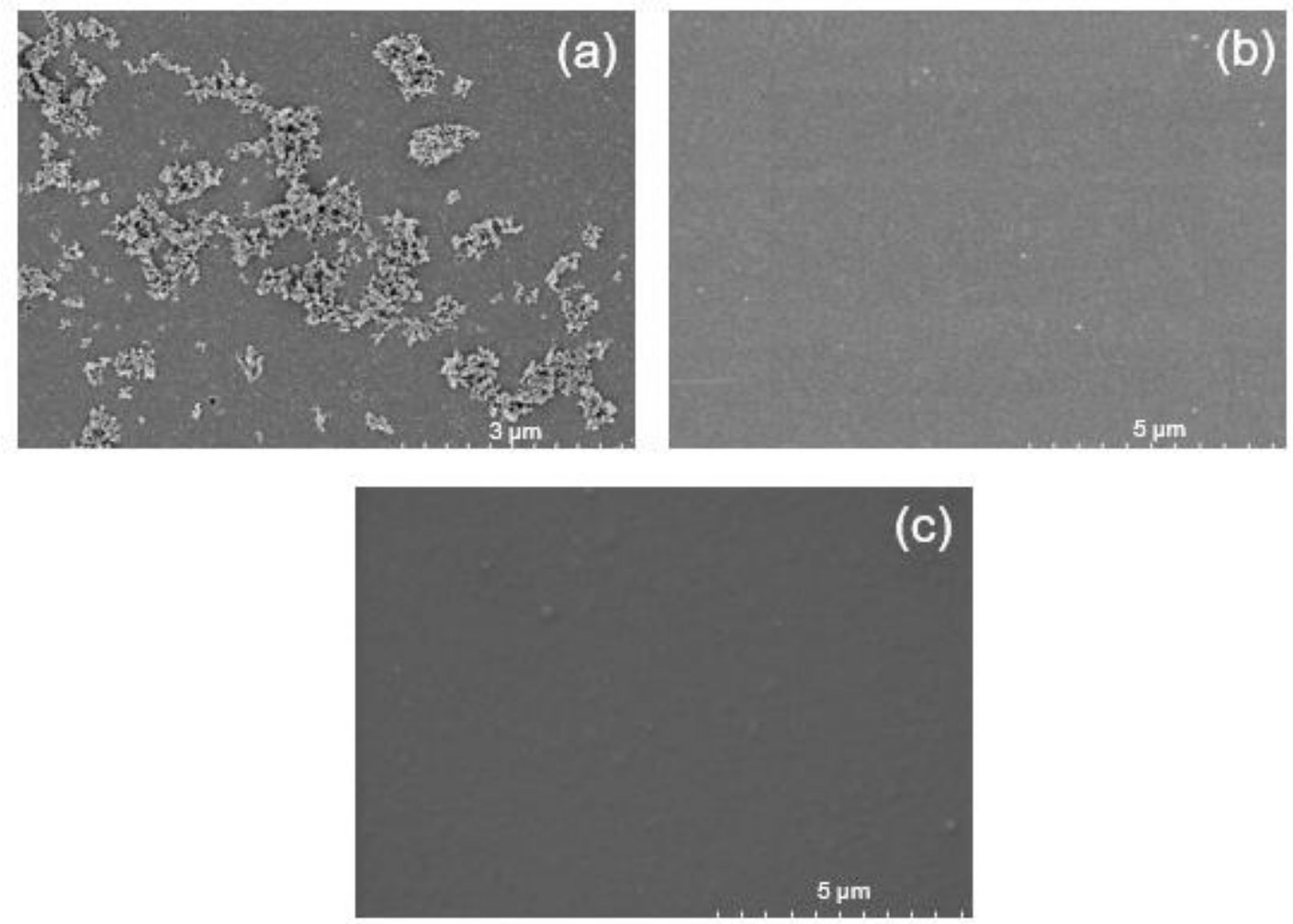

Deactivation of the antigenic sites by desiccation is a concern that has been raised with respect to gasphase immunochemical reactions. The retention of waters of hydration by the immobilized antibody is not disputed, but whether the water activity is sufficient in order for immunochemical reactions to occur has been questioned $[14,15]$. Some have claimed that dry air contains sufficient moisture for immunochemical reactions to occur [14]. In this study, conditioned room air (average $\mathrm{RH}=41.2 \%$ ) was used to convey the powder into the exposure chamber; hence, moisture was always present in the aerosol. The results suggest that water activity of the antibodies is adequate for the selective immunochemical reaction to occur in the gas phase, as suggested previously [12-15].

\subsection{QCM Sensor Response as a Function of Fel d 1 Mass Concentration}

The particle concentration of the cat allergen powder inside the exposure chamber was monitored at sampling intervals of $<1$ min using a particle spectrometer. At higher particle concentrations $\left(>10^{5}\right.$ particles/L), the sampling interval was increased to 5 min due to instrument constraints. The measured particle concentration 
was converted to a mass concentration using Equation (2). Fig. 5 shows that the $\Delta f$ after $30 \min \left(\Delta f_{30} \min \right)$ of continuous allergen addition is a direct function of particle concentration and increases linearly (log-log, $R^{2}=0.968$ ) over a broad concentration range, from $-0.53 \mathrm{~Hz}$ at $5.2 \mathrm{ng} / \mathrm{L}$ to $-8.6 \mathrm{~Hz}$ at $1.6 \times 10^{5} \mathrm{ng} / \mathrm{L}$. The relationship between the $-\Delta f$ and deposited mass per unit area on the QCM crystal $(\Delta m)(\mathrm{ng} / \mathrm{cm})$ is determined using Equation (1), where the sensitivity factor $C_{f}$ is $0.056 \mathrm{~Hz} \cdot \mathrm{cm}^{2} / \mathrm{ng}$ for a $5 \mathrm{MHz}$ crystal at $20^{\circ} \mathrm{C}$.

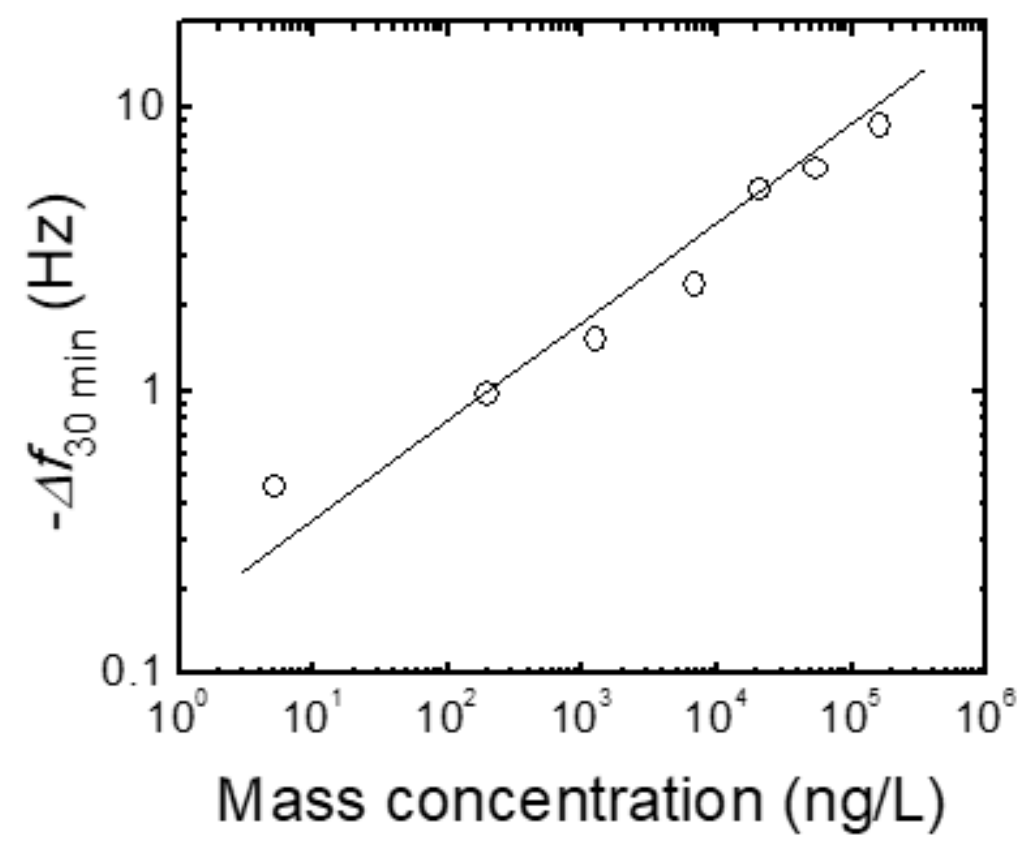

At the minimum detection limit of $5.2 \mathrm{ng} / \mathrm{L}$, the measured $\Delta f_{30} \min$ of $-0.53 \mathrm{~Hz}$ corresponds to a $\Delta m$ of $9.5 \mathrm{ng} / \mathrm{cm}^{2}$. The resolution of the QCM instrument that was used is $<0.4 \mathrm{ng} / \mathrm{cm}^{2}$, which suggests that the sensitivity of the immunosensor could be significantly enhanced. Indeed, the minimum detection limit is the result of a physical limitation of the system: the particle concentration in the exposure chamber was low (50 particles/L) and below this concentration the capture efficiency approaches zero as $f$ shifts cannot be distinguished from instrument drift. The apparatus could be modified to improve capture efficiency in a number of ways. For instance, the flowrate of the carrier gas can be decreased [13,15], the volume of the exposure chamber can be reduced, and the size of inlet tubing can be increased to avoid transport losses. 
Reported airborne concentrations of indoor Fel $d 1$ are typically lower $\left(<0.1-40 \mathrm{ng} / \mathrm{m}^{3}\right)[17,20,21]$ than the lower detection limit of the immunosensor used for this study; however, the Fel $d 1$ powder concentration that was used $(100 \mu \mathrm{g} / \mathrm{g})$ is within the concentration range in settled US house dust as reported by Arbes et al. [18]. Based on the study results, the upper concentration limit of the immunosensor apparatus is $1.6 \times 10^{5} \mathrm{ng} / \mathrm{L}$.

\section{CONCLUSIONS}

A real-time QCM-based immunosensor for airborne cat allergen was developed by immobilization of monoclonal antibodies using a cysteamine SAM. The $f$ shifts as a function of time exhibited Sauerbrey-like behavior when exposed to suspensions of cat allergen powder, which did not require separation of other particles such as dust. Control experiments featured an inert test dust and an E. coli antibody-coated QCM crystal. An increase in $f$ shifts was observed in all negative control experiments, which is indicative of weak interactions with the biofunctionalized surface. A lack of non-specific binding during these control experiments was confirmed using SEM imaging.

The lower airborne allergen concentration limit of the immunosensor was $5.2 \mathrm{ng} / \mathrm{L}$, which is considerably higher than typical indoor Fel $d 1$ concentrations $\left(<0.1-40 \mathrm{ng} / \mathrm{m}^{3}\right)$. However, the lower detection limit was a result of a physical limitation of the experimental apparatus as very low particle concentrations $(<50$ particles/L) resulted in poor aerosol capture efficiency. Modification of the experimental apparatus could enhance sensitivity and resolution of the system. Improvement of immunosensor sensitivity, i.e., the magnitude of the measured $\Delta f$, should be further investigated. This could be realized by using similar piezoelectric devices, such as SAW, which have much higher fundamental operating frequencies. Lower detection limits are also possibly attainable by using powder with lower allergen concentrations. Other concerns, such as activity and stability of the bioaerosol and the antibody-coated crystal during long-term usage 
should also be addressed. This work demonstrates the possibility of a QCM-based immunosensor for airborne cat allergen powder as a sensitive, specific and rapid alternative to conventional techniques of monitoring airborne allergens and other bioaerosols. 


\section{ACKNOWLEDGEMENTS}

This work is supported by the Natural Sciences and Engineering Research Council of Canada (SENTINEL Network), the Fonds Québécois de la Recherche sur la Nature et les Technologies (FQRNT), l'Institut (Robert Sauvé) de Recherche sur la Santé et la Sécurité au Travail (IRSST), the U.S. National Science Foundation Grant Number CBET 0846704, and the U.S. Environmental Protection Agency award EM-83340401-0 via the Syracuse CoE CARTI program. Any opinions, findings, and conclusions or recommendations expressed in this material are those of the authors and do not necessarily reflect the views of the agencies. The authors thank Dr. James Freihaut from The Pennsylvania State University for providing cat allergen powder for the project and Dr. F. Bridier (ETS) for his assistance with SEM. 


\section{REFERENCES}

[1] T. Reponen, K. Willeke, S. Grinshpun, A. Nevalainen, Biological Particle Sampling, Aerosol Measurement, John Wiley \& Sons, Inc.2011, pp. 549-70.

[2] Z. Xu, Y. Wu, F. Shen, Q. Chen, M. Tan, M. Yao, Bioaerosol Science, Technology, and Engineering: Past, Present, and Future, Aerosol Science and Technology, 45(2011) 1337-49.

[3] J. Lee, J. Jang, D. Akin, C.A. Savran, R. Bashir, Real-time detection of airborne viruses on a mass-sensitive device, Appl Phys Lett, 93(2008).

[4] A. Janshoff, H.-J. Galla, C. Steinem, Piezoelectric Mass-Sensing Devices as BiosensorsĐAn Alternative to Optical Biosensors?, Angew Chem Int Ed, 39(2000) 4004-32.

[5] S.-H. Lee, D.D. Stubbs, J. Cairney, W.D. Hunt, Rapid detection of bacterial spores using a quartz crystal microbalance (QCM) immunoassay, Sensors Journal, IEEE, 5(2005) 737-43.

[6] C.D. Earle, E.M. King, A. Tsay, K. Pittman, B. Saric, L. Vailes, et al., High-throughput fluorescent multiplex array for indoor allergen exposure assessment, Journal of Allergy and Clinical Immunology, 119(2007) 428-33.

[7] T.W. Owen, R.O. Al-Kaysi, C.J. Bardeen, Q. Cheng, Microgravimetric immunosensor for direct detection of aerosolized influenza A virus particles, Sensors and Actuators B: Chemical, 126(2007) 691-9.

[8] C. Poitras, N. Tufenkji, A QCM-D-based biosensor for E. coli O157:H7 highlighting the relevance of the dissipation slope as a transduction signal, Biosens Bioelectron, 24(2009) 2137-42.

[9] G.Z. Sauerbrey, The use of quartz oscillators for weighing thin layers and for microweighing, Zeitschrift fuer Physik, 155(1959) 20622.

[10] C. Poitras, J. Fatisson, N. Tufenkji, Real-time microgravimetric quantification of Cryptosporidium parvum in the presence of potential interferents, Water Res, 43(2009) 2631-8.

[11] K.J.M. Sandstrom, A.P. Turner, Biosensors in air monitoring, Journal of environmental monitoring : JEM, 1(1999) 293-8.

[12] D.D. Stubbs, W.D. Hunt, S.H. Lee, D.F. Doyle, Gas phase activity of anti-FITC antibodies immobilized on a surface acoustic wave resonator device, Biosensors and Bioelectronics, 17(2002) 471-7.

[13] J. Ngeh-Ngwainbi, P.H. Foley, S.S. Kuan, G.G. Guilbault, Parathion antibodies on piezoelectric crystals, Journal of the American Chemical Society, 108(1986) 5444-7.

[14] C.K. O'Sullivan, R. Vaughan, G.G. Guilbault, Piezoelectric Immunosensors - Theory and Applications, Analytical Letters, 32(1999) 2353-77.

[15] L. Rajaković, V. Ghaemmaghami, M. Thompson, Adsorption on film-free and antibody-coated piezoelectric sensors, Anal Chim Acta, 217(1989) 111-21.

[16] M. Jenik, A. Seifner, P. Lieberzeit, F.L. Dickert, Pollen-imprinted polyurethanes for QCM allergen sensors, Anal Bioanal Chem, 394(2009) 523-8.

[17] C.M. Luczynska, Y. Li, M.D. Chapman, T.A.E. Platts-Mills, Airborne Concentrations and Particle Size Distribution of Allergen Derived from Domestic Cats (Felis domesticus): Measurements Using Cascade Impactor, Liquid Impinger, and a Two-site Monoclonal Antibody Assay for Fel d I, American Journal of Respiratory and Critical Care Medicine, 141(1990) 361-7.

[18] S.J. Arbes Jr, R.D. Cohn, M. Yin, M.L. Muilenberg, W. Friedman, D.C. Zeldin, Dog allergen (Can f 1) and cat allergen (Fel d 1) in US homes: Results from the National Survey of Lead and Allergens in Housing, Journal of Allergy and Clinical Immunology, 114(2004) $111-7$.

[19] H. Grönlund, J. Adédoyin, R. Reininger, E.M. Varga, M. Zach, M. Fredriksson, et al., Higher immunoglobulin E antibody levels to recombinant Fel d 1 in cat-allergic children with asthma compared with rhinoconjunctivitis, Clin Exp Allergy, 38(2008) 1275-81.

[20] A. Custovic, A. Simpson, H. Pahdi, R.M. Green, M.D. Chapman, A. Woodcock, Distribution, aerodynamic characteristics, and removal of the major cat allergen Fel d 1 in British homes, Thorax, 53(1998) 33-8.

[21] S. Raja, Y. Xu, A.R. Ferro, P.A. Jaques, P.K. Hopke, Resuspension of indoor aeroallergens and relationship to lung inflammation in asthmatic children, Environment International, 36(2010) 8-14.

[22] J. Freihaut, M. Chapman, A. Salapatek, P. Patel, S. McCue, Aerodynamic properties of milled allergen products: preparation of "standard", air-suspendable dog, cat, cockroach and mite allergen powders, Allergy, 62(2007) 38-.

[23] A.L.J. Olsson, H.C. van der Mei, D. Johannsmann, H.J. Busscher, P.K. Sharma, Probing Colloid-Substratum Contact Stiffness by Acoustic Sensing in a Liquid Phase, Analytical Chemistry, 84(2012) 4504-12.

[24] G.L. Dybwad, A sensitive new method for the determination of adhesive bonding between particle and a substrate, J Appl Phys, 58(1985) 2789-90. 
[25] E. Vittorias, M. Kappl, H.J. Butt, D. Johannsmann, Studying mechanical microcontacts of fine particles with the quartz crystal microbalance, Powder Technology, 203(2010) 489-502.

[26] U. Latif, S. Can, O. Hayden, P. Grillberger, F.L. Dickert, Sauerbrey and anti-Sauerbrey behavioral studies in QCM sensorsDetection of bioanalytes, Sensors and Actuators B: Chemical, 176(2013) 825-30. 


\section{FIGURE CAPTIONS}

Figure 1. (a) Number-based and (b) volume-based size distributions for the cat allergen powder. (c) Number-based and (d) volume-based size distributions for the non-allergen test dust.

Figure 2. (a) Flow diagram of the experimental setup. (b) Isometric schematic of the exposure chamber with principal airflow trajectories. The schematic is not drawn to scale.

Figure 3. Representative measured frequency shifts ( $\Delta f$ ) as a function of time for a Fel 1 antibody (Ab)-modified $Q C M$ crystal exposed to test dust (negative control) and different cat allergen powder concentrations, and the measured $\Delta$ f for an E. coli $\neg$ Ab-coated QCM crystal exposed to cat allergen powder at $1.4 \times 103 \mathrm{ng} / \mathrm{L}$.

Figure 4. Representative SEM images of: Fel 1 antibody-functionalized surfaces after (a) 30 min of continuous exposure to cat allergen powder; (b) 30 min of continuous exposure to test dust; (c) E. coli antibody-functionalized surface after 30 min of continuous exposure cat allergen powder. 10,000 $\times$ magnification. $212 \times 77 \mathrm{~mm}(150 \times 150$ $D P I)$.

Figure 5. Average measured negative frequency shift after 30 min of Fel 1 exposure as a function of cat allergen powder particle concentration. 


\section{AUTHORS BIOGRAPHIES}

David R. P. Morris is a graduate student in chemical engineering at McGill University. He received his B.Eng. in chemical engineering from McGill University in 2012. He worked as a research assistant in the Biocolloids and Surface Laboratory at McGill University during 2010 and at the Porous Materials Engineering and Analysis Laboratory at McGill University between 2011 and 2012. His current research interest is in catalysis and gas separation and storage using metal organic frameworks.

Julien Fatisson, $\mathrm{PhD}$, is a research associate at the École de Technologie Supérieure in Montréal, associate member of the RRSSTQ and assistant to the head of the RRSSTQ workplace safety strategic grouping. His research interests include the analysis of liquid phase behaviour and surface deposition of synthetic nanoparticles using quartz crystal microbalance and the study of nanoparticles' toxicity to human cells, as well as the implementation of an adaptive decision-aid tool for evaluating risks associated with nanoparticles.

Adam L. J. Olsson, PhD, is currently a postdoctoral fellow at the department of Chemical Engineering at McGill University in Montreal. He received his M.Sc. degree in Molecular Biology from Gothenburg University in 2006 and his Ph.D. degree in Biomedical Engineering from Groningen University in 2011. His research interests include colloid, surface and interfacial science, bioadhesion and acoustic sensing.

Nathalie Tufenkji is an Associate Professor of Chemical Engineering at McGill University and holds the Canada Research Chair for Biocolloids and Surfaces. She earned the B.Eng. degree in Chemical Engineering from McGill University in 1999, and M.Sc. and Ph.D. degrees in Chemical and Environmental Engineering from Yale University in 2001 and 2005, respectively. Her current research interests include environmental nanotechnology, QCM-D based biosensors, and the health benefits of plant and berry derivatives.

Andrea R. Ferro is an Associate Professor of Civil and Environmental Engineering at Clarkson University. She earned M.Sc. and Ph.D. degrees in Civil and Environmental Engineering from Stanford University in 1992 and 2002, respectively. She is a registered professional engineer with approximately 25 years of experience in field studies, engineering design, and project management in environmental health and engineering. Her technical expertise is focused on indoor air quality and human exposure to airborne pollutants. 